\title{
LA MORALIDAD COMO BASE PARA EL PROCESO DE AXIOMATIZACIÓN
}

\author{
Manuel Asdrúbal Prieto Salas \\ Universidad Católica de Colombia
}

\section{Resumen}

El proceso de axiomatización de conductas humanas mediante el establecimiento de leyes restrictivas puede ser ilustrado por medio de la teoría de la construcción de posiciones morales válidas desarrolladas por Ronald Dworkin; en ella es evidente la presencia de elementos de coherencia entre el sistema de creencias humanas. Estos elementos bien estructurados cimentan una moral válida que, junto con otras de común acuerdo, pueden prohibir conductas que atenten contra la colectividad.

La finalidad del artículo es desvirtuar los postulados defendidos por el positivismo ideológico, el cual rechaza de manera tajante la influencia de la moral pública dentro de un sistema jurídico; para alcanzar dicho fin, es menester elucidar el vínculo entre la moral pública convencional y el sistema normativo como un pacto indisoluble. Mediante este acuerdo, los miembros de una sociedad proscriben conductas específicas, calificables bajo el rótulo de inmorales. Aunque a simple vista es una hipótesis plausible, se complejiza cuando tratamos de aplicarla a una sociedad moderna caracterizada por la pluriculturalidad.

Palabras clave: moral, racionalización, sistema jurídico, pornografía, homosexuales.

El autor: docente de Lógica Jurídica, Teoría del Derecho e Historia de las ideas políticas. Investigador adscrito al Grupo de Estudios Legales y Sociales Phronesis del Centro de Investigaciones Socio Jurídicas (CISJUC) de la Facultad de Derecho de la Universidad Católica de Colombia. Correo electrónico:maprieto@ucatolica.edu.co

Recibido: 26 de julio de 2013; evaluado: 1 de agosto de 2013; aceptado: 25 de noviembre de 2013.

Este artículo se estructura como desarrollo del proyecto de investigación "Conceptuadores operativos en el orden del Derecho", del Grupo de Estudios Legales y Sociales Phronesis, adscrito al Centro de Investigaciones Socio Jurídicas (CISJUC) de la Facultad de Derecho de la Universidad Católica de Colombia.

NOVUM JUS • ISSN: 1692-6013 • Volumen 9 Nº 1 • Enero - Junio 2015 • Págs. 29-49 


\title{
MORALITY AS A BASIS FOR THE AXIOMATIZATION PROCESS
}

\author{
Manuel Asdrúbal Prieto Salas \\ Universidad Católica de Colombia
}

\begin{abstract}
The process of axiomatization of human behavior by establishing restrictive laws can be illustrated by means of the theory of the construction of valid moral positions developed by Ronald Dworkin, in which it is evident the presence of elements of coherence between the system of human beliefs. These well-structured elements underpin a valid moral, along with other common agreement, may prohibit behaviors that jeopardize the community.

The purpose of the article is to undermine the principles defended by the ideological positivism, which flatly rejects the influence of public morals within a legal system for achieving that, it is necessary to elucidate the link between conventional public morality and the regulatory system as an indissoluble bond. Under the agreement, the members of a society proscribe specific behaviors, considered as "immoral". Though superficially this is a plausible hypothesis, it becomes more complex when we try to apply it to a modern society characterized by multiculturalism.
\end{abstract}

Keywords: moral, racionalization, legal system, pornography, homosexuals.

About the author: Professor of Legal Logic, Theory of Law and History of Political Ideas. Researcher at the "Phronesis" Group of Legal and Social Studies, of the Centro de Investigaciones SocioJurídicas (Socio-Legal Research Institute) of the School of Law of the Catholic University of Colombia. E-mail: maprieto@ucatolica.edu.co

Received: July 26, 2013; reviewed: August 1, 2013; accepted: November 25, 2013. 


\section{Introducción}

Hoy en día, las teorías jurídicas que conceptúan el Derecho como un conjunto de normas reguladoras de conducta están desactualizadas, porque no vislumbran características determinantes de todo el sistema como la vinculación existente entre Derecho y moral; este aspecto problemático es uno de los ejes centrales de la conceptuación del Derecho del jurista estadounidense Ronald Dworkin, quien desarrolla una óptica relacional que posibilita una descripción plausible del fenómeno jurídico. Sin embargo, de la aplicabilidad de estas teorías surgen nuevos interrogantes que merecen un análisis cuidadoso.

Uno de los problemas emergentes de mayor peso dentro de la teoría jurídica dworkiniana es la influencia de la moral convencional en el sistema jurídico, con el fin de que este último proscriba y sancione las conductas calificadas como inmorales. La elucidación de este problema requerirá implementar, desde lo metodológico, una investigación filosófica y crítica de las diversas teorías que han tratado de describir este fenómeno, entre las que encontraremos las esgrimidas por Lord Devlin en las polémicas conferencias llamadas "La imposición de la moral", en las cuales ataca la homosexualidad; la teoría de la mutabilidad social de Hart y la influencia que ejerce en la conformación de una convención moral pública; el adensamiento del Derecho, producto de la penetración del sistema jurídico en el mundo de la vida, visto desde la teoría de la acción comunicativa de Habermas; el proceso de axiomatización sistemática de pautas morales por medio de la valuación normativa de las teorías lógico-deónticas de Ulises Schmill y los parámetros argumentativos de calificación moral de posturas.

Esta investigación tiene como propósito desvirtuar los postulados defendidos por el positivismo ideológico, el cual rechaza de manera tajante la influencia de la moral pública en un sistema jurídico; para alcanzar dicho fin, es preciso explicar la relación existente entre la moral pública convencional y el sistema normativo como un pacto indisoluble. Mediante este acuerdo, los miembros de una sociedad proscriben conductas específicas calificables con el rótulo de inmorales. Aunque a simple vista es una hipótesis plausible, se complejiza cuando tratamos de aplicarla a una sociedad moderna caracterizada por la pluriculturalidad. En este tipo de sociedades, sus miembros adoptan diferentes posturas morales que limitan la posibilidad de acuerdo, situación que requiere desarrollar, de manera argumentada, un procedimiento que permita diferenciar cuáles sistemas de creencias son constitutivos

NOVUM JUS • ISSN: 1692-6013 • Volumen 9 Nº 1 • Enero - Junio 2015 • Págs. 29-49 
de una postura moral válida y cuáles son producto de fobias, racionalizaciones o copias de otras posturas.

\section{I}

En la mayoría de las sociedades modernas de tradición occidental, la calificación moral de una conducta humana requiere la utilización de teorías ético-normativas como las teleológicas y deontológicas. Cada una de ellas, dentro de su estructura lógica, da primacía a un operador específico que tendrá como fin determinar si una acción es moral o no. Por ejemplo, en las teorías teleológicas, la idea de bien prima sobre la idea de lo correcto desde la moral. Por el contrario, las teorías deontológicas priorizan la idea de lo moralmente correcto sobre lo bueno. ${ }^{1}$

Las teorías teleológicas están encaminadas a la perfección del ser humano. Esta cualidad se adquiere, sobre todo, al adecuar la conducta del individuo a las prescripciones morales emitidas por Dios. Las transgresiones a estas leyes divinas son inmorales, no porque Dios las prohíba, sino que Dios las prohíbe porque son incorrectas, es decir, la inmoralidad de las acciones humanas no derivan de la arbitrariedad del todopoderoso. ${ }^{2}$

Por otra parte, dentro de las teorías deontológicas de determinación moral, la esbozada por Dworkin ${ }^{3}$ merece una especial atención, ya que pretende construir un proceso argumentativo de axiomatización, que posibilita la inclusión de pautas morales. Plantea una pregunta ineludible sobre esta temática: ¿qué consecuencias para el sistema jurídico puede generar la calificación de una conducta como inmoral? Y, si es etiquetada de ese modo, ies suficiente para que el sistema la prohíba la conducta por medio de un mandato $?^{4}$

\footnotetext{
Carlos Nino, Introducción al análisis del Derecho (Barcelona: Ariel, 1983), 383.

Santo Tomás de Aquino, La suma de Teología II parte I-II (Madrid: Biblioteca de autores cristianos, 1993), 552-556.

3 Ronald Dworkin, Los derechos en serio (Barcelona: Ariel, 2007), 349.

4 Hay que tener en cuenta que el mandato es una figura amplia y sencilla en la que está inmerso el concepto de ley, es decir, las leyes son una clase de mandato que consiste en la manifestación de un deseo dirigido a una persona para que esta haga u omita una acción y, en caso de incumplimiento, la expone a una sanción. Así, las órdenes emitidas en un lenguaje imperativo que no anexan una sanción por incumplimiento no pueden ser consideradas mandatos. John Austin, El objeto de la jurisprudencia (Madrid: Centro de Estudios Políticos y Constitucionales, 2002), 37-41.
} 
La problemática moral sobre la conducta humana ha tenido un rico desarrollo en diversas ciencias sociales distintas al Derecho, como la Sociología y la Filosofía. Sin embargo, el hecho de que las conductas puedan ser asimiladas por un sistema jurídico tras procesos de axiomatización, exige que se aborde este tema desde el punto de vista jurídico, puesto que el proceso de axiomatización utilizado por una autoridad nomotética para transformar una conducta inmoral en una conducta proscrita por la ley es bastante complejo. La autoridad nomotética es la facultad de una entidad con poder político de influir en la conducta de sujetos normativos de distinto nivel; dichos niveles de influencia son determinados por el destinatario de la ley. ${ }^{5}$

Desde el punto de vista jurídico, pueden surgir cuestiones problemáticas referentes a conductas que popularmente son encasilladas como inmorales, por ejemplo, la pregunta respecto al papel del rechazo público: si en un sistema jurídico se aceptara como una razón plausible para la configuración de prohibiciones jurídicas, ${ }^{6}$ se vulnerarían principios fundamentales aceptados por todo sistema jurídico moderno. Si dentro de un corpus normativo ${ }^{7}$ están contenidos el principio universal de la libertad individual y a la vez una norma de orden superior que valida la opinión pública como fundamento de producción normativa, en algún momento se presentará un error de concurrencia paralela que lo hará incoherente y falible. Por consiguiente, todo ordenamiento jurídico que da prelación a las decisiones de la masa termina por limitar todo principio o derecho de orden individual o personal de sus afectados.

Para elucidar esta problemática, Dworkin utiliza la teoría jurídico-moral desarrollada por Lord Devlin, quien en 1958 presentó una conferencia titulada "La imposición

5 Por ejemplo, en la teoría normativa de orden superior del sistema lógico deóntico de Georg Henrik Von Wright, se manejan dos modus u operadores lógicos: el de obligatoriedad $(\mathrm{O})$ y el de permisión $(\mathrm{P})$. A estos operadores se les anexa una letra minúscula (p, q, r, s, t, u, v) que simboliza el contenido ordenado o permitido en la norma. A diferencia de otras, las normas de orden superior se caracterizan por tener dos operadores deónticos seguidos de una variable OOp (debe ser el caso que se haga obligatorio el caso de que p), PPp (está permitido que se permita el caso de que p), OPp (es obligatorio que permita el caso de que p), cuya característica más importante es que son el desarrollo del principio de transferencia de voluntad, en el cual una norma superior ordena o permite a una autoridad subordinada que funge como agente en una ocasión específica para que produzca una obligación o una permisión, la satisfacción de la norma de orden superior OOp no se agota con el cumplimiento de la autoridad subordinada al producir Op, sino que el estado de cosas que esta última obliga o permite que se haga efectivo, es decir, que el estado de cosas p suceda. Georg Henrik Von Wright, Normas, verdad y lógica (México D. F: Fontamara, 2001), 30-32.

Dworkin, Los derechos en serio, 349.

El corpus normativo alude al conjunto de normas que guardan una relación de coherencia. Para su satisfacción requieren que se satisfaga cada una de las normas consagradas en él. Si por alguna razón no se satisfacen, el corpus tampoco lo hará. En la vida real es claro que las normas se vulneran, pero eso no indica que el corpus sea incoherente e irracional. La racionalidad de las normas hace referencia a que la voluntad del legislador está destinada a que dichas normas se cumplan. Von Wright, Normas, verdad y lógica, 37-40.

NOVUM JUS • ISSN: 1692-6013 • Volumen 9 Nº. 1 • Enero - Junio 2015 • Págs. 29-49 
de la moral", ${ }^{8}$ en la cual fundamentaba la necesidad de toda sociedad de prohibir, de manera tajante, las prácticas homosexuales, con base en el siguiente argumento: si la sociedad se despoja de todos sus prejuicios y ve a una determinada conducta como una abominación, entonces no puede negársele el derecho de castigar dicha conducta. Según el jurista estadounidense, Lord Devlin estructuró dicha aseveración desde el utilitarismo y afirma que la función del Derecho es salvaguardar el orden público y la decencia y que no le son atribuibles otras funciones como la de intervenir en la vida privada de los ciudadanos ni imponer modelos de vida.

En este modelo existe un ámbito de inmoralidad y moralidad privada que no son asuntos del Derecho, mas dicho principio utilitarista no es aplicable al Derecho Penal. Este cambio de parecer tan abrupto, dentro del sistema devliniano, empieza a desmoronar poco a poco sus bases filosóficas utilitaristas, porque, según el autor, el Estado desempeña un rol de tutor moral de sus asociados y la técnica más idónea para lograr dicha labor es el Derecho Penal. Es claro que este último argumento no guarda coherencia ni compatibilidad con un Derecho Penal de corte utilitarista en el cual la sociedad, por mayoría, puede calificar una conducta como delito, si esta es desaprobada por sus miembros, incluso si no representa una lesividad intersubjetiva. ${ }^{9}$ El cambio de perspectiva de Devlin se fundamentó en dos grandes argumentos: el primero, sobre el derecho de la sociedad de proteger su existencia y, el segundo, sobre el derecho de la mayoría a seguir sus convicciones morales y defender su medio social de aquellos cambios a los que se opone. El argumento inicial parte de que en toda sociedad existen dos tipos de principios morales. El primero es aquel cuyo obedecimiento es libre y voluntario y no se puede imponer por ningún medio coercitivo; en este evento, como miembro de una comunidad con una organización jurídica, el ciudadano estructura los imperativos que regularán su comportamiento intersubjetivo de acuerdo con dicho principio moral, sin que medie algún tipo de represión, aunque existen estándares morales de obligatorio cumplimiento. En caso de transgresión e inobservancia, es necesario que haya un medio coercitivo, porque para sobrevivir y subsistir una sociedad debe tener como mínimo unos modelos morales impositivos que se edifican en un consenso moral; por esta necesidad de subsistencia de las sociedades, ellas adquieren el derecho a sobrevivir. ${ }^{10}$

Dworkin, Los derechos en serio, 350

Dworkin, Los derechos en serio, 351.

10 Es claro que para Lord Devlin, en su primer argumento, cualquier conducta tendiente a contravenir dichos estándares morales adoptados por consenso afecta de manera directa y flagrante contra la vida de la sociedad; así, es admisible que esta última, en defensa de su supervivencia, axiomatice y asimile dichas conductas para prohibirlas y así garantizar la unidad moral, que es el sustento de su vida. Dworkin, Los derechos en serio, 355 . 
En virtud de ese derecho a la vida que adquiere la sociedad, ella queda facultada para garantizarlo por todos los medios que tenga a disposición. Así, los medios más idóneos son las instituciones estatales y el Derecho Penal. Este último, en particular, es la mejor herramienta para salvaguardar dichos estándares morales consensuados, que son los elementos básicos que mantienen con vida a una sociedad.

Un rasgo curioso de este primer argumento es que la sociedad puede utilizar el Derecho para ser mucho más estática y menos mutable; esta perturbadora característica se extrae de una afirmación suya, acerca de que es correcto instrumentalizar el Derecho para evitar una corrupción del consenso que mantiene unida a la sociedad. Si concibiéramos un sistema de Derecho estructurado a la manera de Lord Devlin, entenderíamos el consenso de cohesión social como un corpus de principios morales que todos hemos convenido y adoptado, pero esta teoría ignora importantes conclusiones a las que han llegado la historia y la Sociología. Una de ellas es que la mutabilidad es un elemento sustancial y esencial de lo que denominamos sociedad y trae varias consecuencias, entre ellas, que los principios morales sociales que hemos consensuado de manera intersubjetiva van perdiendo eficacia en el sistema de creencias de nuevas generaciones y que estas, de acuerdo con su realidad, reemplazan estos viejos principios en desuso por otros con mayor aplicabilidad al fragmento espacio-temporal en donde están ubicados, con lo que se crea un nuevo corpus. Este proceso social es inconcebible desde las razones que estructuran este primer argumento esgrimido por Devlin. ${ }^{11}$

El poder de castigar actos considerados inmorales por trasgredir estándares morales convencionales no puede ser absoluto, toda vez que, en cada sociedad, debe existir una barrera protectora que restrinja este poder. Sobre este tema de límites y órbitas de no injerencia estatal en el principio universal de la libertad, Immanuel Kant ${ }^{12}$ plantea un concepto similar en La metafísica de las costumbres, cuando afirma que el Derecho establece las condiciones mínimas en donde el arbitrio individual puede ser compatible con el arbitrio de los demás, pero las teorías tienen un punto donde no existe compatibilidad. Para Kant, las condiciones mínimas que otorga el Derecho regulan la relación individuo-individuo que se extienden con cualquier miembro de la sociedad a la que pertenecen, mas no alude a una relación individuo-sociedad y mucho menos adjudica características de ser vivo a la sociedad, que tiene una necesidad de subsistencia básica que la faculta para atropellar a la individualidad de

11 Dworkin, Los derechos en serio, 352.

12 Immanuel Kant, La metafisica de las costumbres (Madrid: Tecnos, 2002), 37-43.

NOVUM JUS • ISSN: 1692-6013 • Volumen 9 Nº. 1 • Enero - Junio 2015 • Págs. 29-49 
uno de los miembros. ${ }^{13}$ En términos del primer argumento, el arbitrio del individuo, dado por el principio universal de la libertad, no debe guardar coherencia con el de otros sujetos, sino estar en consonancia con los estándares morales que maneja la sociedad. En caso de incompatibilidad, la sociedad queda facultada para imponer dichos principios por medio del Derecho Penal; además, existe la posibilidad de infligir un daño a la persona que ha transgredido dichos estándares.

Otra discrepancia de peso entre las dos teorías se refiere a la legitimación de la coacción. Para Devlin se justificaría en el sentido de una necesidad manifiesta de autopreservación por parte de la sociedad, entendida como un ser viviente; Kant justifica la coacción como un límite de la libertad desbordada de otros, ya que si se permitiera ese desbordamiento, la órbita de libertad de otras personas se afectaría de manera radical. En ese sentido, la coacción armoniza y equilibra las libertades individuales para la convivencia pacífica. ${ }^{14}$

Los principios restrictivos obligan a la sociedad a ser más cautelosa para calificar cierta conducta como inmoral, lo que imposibilita al Derecho para tratarlas, pero la sociedad puede romper con facilidad el dique de los derechos restrictivos, cuando dicha conducta inmoral llega a ser "intolerante, indigna o repugnante" ${ }^{15}$ En otras palabras, si a una conducta se le califica con estos adjetivos, la sociedad tiene el pleno derecho de prohibirla y rotularla como delito. En el marco interpretativo esto es muy claro y es razonable concluir que las condiciones sexuales diferentes, como la homosexualidad o los gustos que no son bien vistos desde lo popular, como el consumo de pornografía fuerte, usar el cabello de colores o tatuarse, podrían sancionarse por medio de mandatos, si llegaran a ser insoportables y repugnantes ante los consensos morales, que son el corazón y el sustento de una sociedad basada en la moralidad pública. Este concepto emerge de la ruptura filosófica del utilitarismo de la construcción conceptual devliniana, que luego construyó un concepto de Derecho y sociedad mediante un sistema de principios morales, denominados "moral pública" y solo en el evento de que ella se vea amenazada, entra en peligro la existencia de la sociedad misma; para evitarlo, la sociedad debe responder con el derecho sancionador para protegerse.

Hart considera que el núcleo esencial de la explicación ofrecida por Devlin es débil y carente de coherencia, porque la concepción que maneja de la sociedad es confusa

\footnotetext{
Kant, La metafísica de las costumbres, 42.

Kant, La metafísica de las costumbres, 41.

Dworkin, Los derechos en serio, 353.
} 
debido a que la sociedad, como un elemento humano convencional, tiene buena estabilidad y es absurdo pensar que cualquier conducta inmoral repugnante, intolerante o indigna amenace su existencia. El funcionamiento de la moral convencional, desde la óptica de Hart, alude a pautas y criterios de comportamiento aceptados en la sociedad que se contraponen a los principios y las reglas morales de un individuo que no comparte el ideal moral general. La construcción de un sistema moral convencional aceptado con amplitud debe tener, como elemento constitutivo, unas reglas primarias de obligación. Este corpus de reglas, como sistema regulador de conducta humana, difiere de otros tipos de corpus en la seriedad con que ejerce su control represivo y el sacrificio y la limitación de la libertad de acción a los que se ven abocados los sujetos normativos que aceptan las reglas de ese sistema. ${ }^{16}$

En estado primitivo, es posible que el individuo regulara sus conductas solo con reglas primarias de obligación moral; sin embargo, la evolución de los Estados trae consigo la necesidad de reglas secundarias de reconocimiento, adjudicación y cambio, cuya función primordial será distinguir cuáles reglas son jurídicas y cuáles no. Este proceso no implica el desconocimiento de las obligaciones emanadas de otros sistemas reguladores de conducta como la moral o el cultural, porque en las sociedades modernas todos ellos funcionan de modo paralelo. ${ }^{17}$

Hart evidencia el error que siempre estuvo presente en el primer argumento. En él, la sociedad se concibe como un elemento artificial, que consiste en un cúmulo de ideas y actitudes morales destinadas a sostener la unidad de todos sus miembros. Por tanto, es intolerable que cada uno de los estatus morales que la conforman tenga el derecho de preservar por la fuerza su precaria existencia. Frente a esta crítica, Devlin replica a Hart que él no considera que cualquier desviación de moral o cualquier actividad subversiva conmine la vida de la sociedad, sino que las dos actividades amenazan la existencia de la sociedad, por tanto no pueden estar más allá del alcance del Derecho. ${ }^{18}$

Al retomar los argumentos de Lord Devlin, sobresale una contradicción argumentativa interna, puesto que al afirmar la existencia de una inmoralidad y una moralidad privadas; en esta última, el Derecho no tiene injerencia. La protección es de carácter constitucional y es desarrollada por los principios restrictivos cuyo

Herbert Hart, El concepto del Derecho (Buenos Aires: Abeledo-Perrot, 1968), 210.

Hart, El concepto del Derecho, 211.

Dworkin, Los derechos en serio, 353

NOVUM JUS • ISSN: 1692-6013 • Volumen 9 Nº 1 • Enero - Junio 2015 • Págs. 29-49 
propósito es salvaguardar el principio universal de la libertad, pero cuando replica a Hart, él mismo contradice su construcción argumentativa, en particular, la barrera constitucional desarrollada por principios restrictivos, ya que es posible que una práctica inmoral dentro de esa órbita de libertad afecte y cuestione la moral pública y, si esto sucede, por lógica se colige que la existencia de la sociedad está en peligro. Al seguir este razonamiento es necesario que el Derecho traspase las barreras de la órbita de la libertad privada y sancione al ciudadano que llevó a cabo tales prácticas. Por otra parte, Dworkin expresa que es una función importante del Derecho resguardar la órbita de la libertad de arbitrio. La construcción jurídica de estas barreras salvaguarda al individuo de la histeria pública temporal. ${ }^{19}$

\section{II}

El segundo argumento alude al derecho de la sociedad de seguir sus propias luces. Así, existe una necesidad tajante de erradicar ciertas conductas porque atentan contra estructuras sociales básicas. Bajo este argumento, permitir la homosexualidad desembocaría en la destrucción de la institución familiar como la entendemos hoy en día, pero esto no le da a la sociedad el derecho de prohibir las prácticas homosexuales por no estar acorde con ciertas costumbres. Sobre este punto es necesaria la intervención del legislador en la determinación de cuáles instituciones sociales, consensuadas desde la moral, deben quedar intactas. Esto significaría que las personas que no se amolden a sus prescripciones tendrían que pagar con su libertad.

Para que un trasgresor pague con su libertad individual, el legislador debe decidir de manera previa si este tipo de acciones es inmoral y si, en efecto, es así, no cuenta la libertad del individuo, toda vez que con este principio universal prefirió entregarse de forma voluntaria y libre a la inmoralidad. ${ }^{20}$

Al usar la moralidad consensual como un sistema de control social, la opinión pública puede prescribir normas de obligación (Op), de permisión (Pp) y de pro-

\footnotetext{
19 Si analizamos con detenimiento, H. L. A. Hart propone un sistema jurídico bastante peligroso y retrógrado, en el que el Derecho positivo sancionador tiene como base de axiomatización la opinión pública y, si dicha opinión no tolera una actividad determinada, esta es razón suficiente para penalizarla bajo el pretexto de que la actividad puede amenazar la existencia de la sociedad misma. Así, toda histeria pública podría punir cualquier actividad que en algún momento le parezca desagradable; este sistema, desde esas relaciones lógicas, es por completo inestable y está destinado a desaparecer por uno de los términos que más ataca Lord Devlin: la revolución. Otra falencia de esta construcción es que ninguna sociedad moderna se ve amenazada por la práctica de conductas repugnantes e impopulares. Dworkin, Los derechos en serio, 354.

20 Dworkin, Los derechos en serio, 356.
} 
hibición (Php) encaminadas a influir en la conducta del sujeto normativo, cuyo cumplimiento depende de la voluntad del sujeto; sin embargo, en caso de transgresión de alguna norma moral, el máximo castigo aplicable es el rechazo social. La intervención del órgano legislador es necesaria para elevar el estatus de estas normas morales inexigibles en lo coercitivo a normas genuinas protegidas por el poder sancionador del Estado.

Como todos los operadores que funcionan en cualquier lógica modal se definen entre sí, deónticamente se puede expresar una obligación en términos de prohibición o en términos de permisión, además del uso del término de enlace negación (-). Una norma que obliga el caso de que $\mathrm{p}(\mathrm{Op})$ es equivalente a la norma que prescribe "no está permitido el caso de que no p" (-P-p) y a la norma que prohíbe el caso de que no $\mathrm{p}$ (Ph-p). Contrario sensu, una norma que prohíbe un estado de cosas (Php), en términos de obligación se expresa "debe ser el caso que no p" (O-p) y en términos de permisión, "no está permitido que p" (-Pp).

La importancia de conocer las interdefinibilidades de las normas radica en que, si pasan por el proceso de valuación, sus equivalencias también serán parte del corpus; las consecuencias de este ejercicio no deben ser tomadas a la ligera, pues si se prohíbe la equivalencia de una norma admitida, el sistema jurídico se torna insatisfactorio y de ella se colige su derrotabilidad. ${ }^{21}$

Este proceso lógico, que es estándar a toda teoría de Derecho y a todo sistema jurídico, consiste en elevar un radical deóntico o norma moral a una norma perteneciente a un sistema jurídico; este estatus se adquiere cuando pasa por un proceso de valuación normativa dado por la función $\mathrm{V}(\mathrm{Op}) .{ }^{22}$ Los criterios de valuación son los que variarán según el sistema legal y el de creencias. Por ejemplo, si usamos el segundo argumento de Devlin, el legislador debe intervenir para proteger la preservación de ciertas instituciones sociales, porque es el derecho de toda sociedad a seguir sus luces. En ese evento, el criterio de valuación normativa en el que se elevará un radical deóntico que protege una institución es el derecho de la sociedad a la autopreservación de costumbres morales consensuadas. A continuación se relaciona cómo se realiza el proceso de axiomatización de prohibiciones que no tienen el estatus normativo.

21 Delia Echave, Ricardo Guibourg y María Urquijo, Lógica, proposición y norma (Buenos Aires: Astrea, 2008), 123.

22 Ulises Schmill, Lógica y Derecho (México D. F.: Fontamara, 2008), 36-38.

NOVUM JUS • ISSN: 1692-6013 • Volumen 9 Nº 1 • Enero - Junio 2015 • Págs. 29-49 
- $\quad \operatorname{Php}(\mathrm{r})$ : radical deóntico que prescribe la prohibición de actos homosexuales.

- V(Php): proceso de valuación en donde la autoridad nomotética estudiará bajo sus criterios si dicha prohibición formará parte del corpus jurídico.

- $\quad \operatorname{Php}(\mathrm{d})$ : norma genuina que ha pasado por el proceso de valuación que ahora pertenece al corpus de un sistema legal.

A estas alturas surge una pregunta sustancial: ¿qué criterios debe tener el legislador para transformar una conducta inmoral en una prohibición legal? Lord Devlin afirma que si la mayoría de la comunidad coincide en una respuesta a estas cuestiones, aun cuando una reducida parte de intelectuales esté en desacuerdo, es deber del legislador guiar sus acciones conforme a dicho consenso. El deber del legislador de acatar la postura de la mayoría se basa en dos razones relacionadas:

1) El último análisis debe hacerse en algún artículo de fe moral y el otro de acuerdo con los principios democráticos.

2) La comunidad debe actuar con sus propias luces, con base en la convicción moral de sus miembros, ya que al fin de cuentas ella es la que trae el Derecho Penal a colación.

Las ideas plasmadas en los dos argumentos utilizados en la réplica a H. L. A. Hart están plagadas de errores: la apreciación que tienen los argumentos sobre la sociología moral son inexactos y simplistas, porque trata de simplificar un fenómeno social de control tan complejo como la moral pública, donde predomina una opinión pública consensuada de lo que es moral o inmoral, aunque la moral, en sí misma, no es similar a las opiniones de una muchedumbre indignada, sino un subsistema social mucho más complejo. ${ }^{23}$

El poder conceptuar un término tan complejo como "moral convencional" no es una tarea fácil, porque su sola definición no abarcaría todas las dimensiones implicadas en él y se hace necesario recurrir a su dimensión semántica y su dimensión pragmática. Si se habla desde la semiótica, la dimensión semántica no agota la descripción global de este fenómeno, por lo que es preciso conocer cómo se relacionan los intérpretes con el fenómeno que están enfrentando ${ }^{24}$.

Dworkin, Los derechos en serio, 358

24 La semiótica es la disciplina encargada del estudio de los signos. Los signos son la base del lenguaje y pueden ser cualquier objeto físico perceptible por los sentidos: una bandera roja, un mapa, una nota escrita, una palabra; todo signo hace referencia a algo, por ejemplo, la bandera roja se relaciona con una carnicería. A 
Para Dworkin, cuando nos referimos a "moral, posición moral, ciencias morales o convicciones morales" desde la pragmática, hablamos del sentido antropológico y son las actividades humanas las encaminadas a la corrección de la conducta bajo las cualidades y sus objetivos humanos. Las expresiones "convicción moral" y "posición moral" también se usan en un sentido discriminatorio cuando son contrastadas con posiciones que implican prejuicios, posturas personales, cuestiones de aversión, gustos personales, actitudes arbitrarias y cosas semejantes. Estas posiciones son evidentes cuando aplicamos cuestiones morales de contenido dudoso para justificar una perspectiva subjetiva. ${ }^{25}$

Las posiciones morales diversas son parte de la moralidad convencional que toda persona comparte. Dentro de este marco de moralidad convencional existe la posibilidad de que, además de nuestras convicciones, haya otras opuestas a las nuestras y que por el hecho de ser morales deben ser respetadas, así no las compartamos. Si dicha posición que aparenta ser moral irrespeta una regla básica del razonamiento moral, no tenemos el deber de respetarla. Sentados estos preceptos, es evidente que la moral convencional delimita cuáles posiciones pueden ser consideradas válidas desde el punto de vista moral y cuáles son las transgresoras de las reglas del razonamiento moral básico.

El proceso mediante el cual la idea de moralidad convencional califica la moralidad o inmoralidad de las posiciones subjetivas requiere una razón que la fundamente; esto no quiere decir que se deba expresar con claridad algún principio moral teórico al que la persona se ajusta. En otras palabras, ella puede desarrollar y mantener una posición moral válida, aunque se ignoren por completo las posturas filosóficas que la alimentan, ya que de manera subjetiva y libre la adoptó como propia porque llenaba sus expectativas. Bajo este entendido, el proceso interno que sirve para tomar una posición moral válida solo requiere una satisfacción subjetiva. ${ }^{26}$ Por otro

lo que hace referencia el signo se llama designado D. Otra característica de los signos es que esa referencia va dirigida a alguien llamado intérprete I; entonces, el proceso semiótico puede definirse de la siguiente manera: $\mathrm{S}$ es signo de D para I, si I piensa en D cada vez que está en presencia de S. Este proceso semiótico tiene tres dimensiones: 1) dimensión sintáctica; 2) dimensión pragmática, y 3) dimensión semántica. La primera se refiere a la relación entre signo y signo como la que existe entre las notas musicales plasmadas en un pentagrama; la segunda, a la relación entre signo e intérprete, por ejemplo, los usos diversos del lenguaje que le dan ciertas culturas a los mismos signos; el tercero, a la relación entre signo y designado, por ejemplo, el sonido de una sirena significa que una ambulancia está cerca. Alicia Gianella de Salama y Margarita Roulet, Lógica simbólica y elementos de metodología de la ciencia (Buenos Aires: El Ateneo, 1986), 3-4.

25 Dworkin, Los derechos en serio, 358.

26 Dworkin, Los derechos en serio, 359.

NOVUM JUS • ISSN: 1692-6013 • Volumen 9 Nº. 1 • Enero - Junio 2015 • Págs. 29-49 
lado, cuando el individuo pensante y libre advierte algún rasgo de una conducta que, desde su punto de vista, es reprobable y puede manifestar su desaprobación de forma razonable, su posición moral es válida. Este ejercicio que recubre de racionalidad su postura frente a un determinado grupo de acciones requiere que sus argumentos sean tamizados por el filtro de los criterios generales de las razones que no cuentan:

1) Primer criterio: si basamos nuestra posición sobre argumentos prejuiciosos. Los prejuicios son posturas fundadas en consideraciones que, por convención, han sido rechazadas por la mayoría de las sociedades. En el caso colombiano, dicho principio fue axiomatizado en el Artículo 13 de la Constitución Política que prescribe que todas las personas nacen libres e iguales ante la ley, recibirán la misma protección y trato de las autoridades y gozarán de igualdad de derechos, libertades y oportunidades sin discriminación por razones de sexo, raza, origen nacional o familiar, lengua, religión, opinión política o filosófica.

2) Segundo criterio: si en el sistema de creencias de un sujeto existe repulsión y asco hacia un grupo de personas o si el sujeto experimenta estas emociones hacia conductas específicas, es necesario revisar las bases de ese sistema de creencias. Si evidenciamos que su repulsión es producto de una reacción emocional, dicha postura no es válida como posición moral. Por esta razón, es preciso distinguir perspectivas morales de reacciones emocionales. Una distinción clara radica en que las primeras justifican de manera racional y convencional a las segundas, pero no ocurre a la inversa. Si el individuo no puede justificar esa emoción desde la razón para que la reconozcamos como una postura moral, dicho sentimiento será considerado como una fobia u obsesión irracional.

3) Tercer criterio: en él se fundamenta la posición moral en una posición de hecho que, además de ser falsa, no es plausible, porque va en contravía de las normas de verificación y discusión que, como mínimo, han sido aceptadas por la sociedad; así, los argumentos ofrecidos por la persona que busca justificar su actuación se reducen a una racionalización desaprobada.

4) Cuarto criterio: si basamos la justificación de una perspectiva moral en sistemas de creencias ajenas como "es que todo el mundo sabe que la pornografía es mala", esta quedará por completo desvirtuada. Este criterio tiene una excepción cuando los argumentos ajenos provienen de consideraciones 
religiosas o divinas. En este evento, la justificación es plausible y tiene validez moral. $^{27}$

\section{III}

La configuración de lo que podemos denominar posición moral es mucho más compleja, porque no basta con la satisfacción de los cuatro criterios dworkinianos sobre las razones que no cuentan. Además, la justificación debe ser coherente con la postura que se adopta o en la que se apoya; por ejemplo, si X justifica su aparente posición moral de rechazo tajante al consumo de carne con el argumento de que atenta contra la vida de los animales, pero compra tenis de cuero de canguro porque están a la moda, es evidente que su sistema de creencias no puede ser calificado como una visión moral válida, pues padece de incoherencia lógico-argumentativa.

Tal incoherencia es extensible a las posiciones basadas en dogmas religiosos. Paralelas al sistema jurídico existen diversas estructuras que regulan la vida del hombre, entre ellas, la religión.

El método para descubrir cuáles son las prescripciones religiosas que regularán al sujeto es el utilizado por Hans Kelsen en la construcción de su dinámica jurídica, la cual presenta dos tipos de sistemas paralelos y complementarios, que son el estático y el dinámico. En el estudio de la moral como ordenamiento normativo de conducta distinto del Derecho, es obligatorio atender al principio estático, toda vez que este es aplicable al sistema de regulación de conductas; contrario sensu, el sistema dinámico tiene como finalidad determinar cuándo se puede rotular a un corpus de normas como jurídico. El primero de estos sistemas pretende explicar el modo en que una norma de orden superior o norma fundante básica "Grundnorm" de corte prescriptivo funciona como fuente de validez del contenido de una serie de normas que se infiere por lógica, es decir, dicha norma fundante se asemeja en un silogismo a la premisa mayor, la premisa menor es una situación fáctica del mundo real y la conclusión es una prescripción técnica. ${ }^{28}$

En la mayoría de los sistemas morales encontramos normas axiomáticas de tipo "es obligatorio decir siempre la verdad"; de esta norma fundante se derivan prescripciones prohibitivas como a) está prohibido mentir, b) está prohibido levantar

27 Dworkin, Los derechos en serio, 361.

28 Hans Kelsen, Teoría pura del Derecho (México D. F.: Dirección de publicaciones UNAM, 1986), 201.

NOVUM JUS • ISSN: 1692-6013 • Volumen 9 Nº 1 • Enero - Junio 2015 • Págs. 29-49 
falsos testimonios, etc. Como la validez de estas normas técnicas se infiere de una de orden superior, es necesario que su contenido sea coherente con el contenido de ella. En caso de que una de orden inferior contradiga a su fundante, esta perderá validez y será inaplicable como parte de ese sistema de control humano. ${ }^{29}$

Una persona que adopta un sistema religioso como la fuente de las razones de sus posiciones morales también debe mantener una relación racional de coherencia entre sus posturas, por ejemplo, con base en la autoridad bíblica, $\mathrm{X}$ condena a todos los homosexuales porque van en contra de las prescripciones de Dios, pero cuando se le pregunta qué opinión tiene sobre las personas que fornican manifiesta que contra ellos no tiene ningún problema, ya que todo el mundo es libre en sus acciones. En este caso, X no justifica su postura con argumentos; es más, está plagada de contradicciones, por lo que no puede explicar de manera razonable que acepta la autoridad bíblica como fuente primigenia de su pensar y actuar; así, su posición moral es incongruente y conlleva a un prejuicio irremediable. ${ }^{30}$

En este punto salen a flote las problemáticas del segundo argumento de Lord Devlin cuando afirma que los legisladores que deben decidir una cuestión moral deben ajustar su consideración a un consenso moral cualquiera; lo que importa es que se sancione una conducta que pueda destruir un orden social positivo. La tesis pierde credibilidad porque el consenso moral no es una posición moral defendible, entendida bajo los cuatro parámetros antes referenciados. ${ }^{31}$

La única posición moral evidente en el segundo argumento es la antropológica: un hombre razonable cree que una conducta es inmoral y ningún miembro de su sociedad puede pensar de otra manera respecto a ella. Por tal razón, es concluyente que la acción sea calificada como inmoral y, por ende, el Derecho adquiere la capacidad de axiomatizarla y prohibirla. Esta postura es un corolario lógico y razonable, si la base de nuestros argumentos es una concepción de posición moral en la que la moralidad es entendida como una mezcla de costumbre y convicción, de razón y sentimiento, de experiencia y prejuicio. No obstante, la moralidad es mucho más que eso; la posición moral no es algo que se pueda conceptuar tan a la ligera, ya que podríamos cimentar un sistema de creencias solo de prejuicios, que nos impedirían vivir en un mundo pluricultural. Asimismo, toma suma importancia la aplicación de criterios de inmoralidad antes de adoptar una postura válida,

Kelsen, Teoría pura del Derecho, 202-204.

Dworkin, Los derechos en serio, 363.

Dworkin, Los derechos en serio, 365. 
para evitar problemas como la racionalización "al crear la falsa idea de que, por sus gustos, algunas personas pueden ser clasificadas como seres inferiores desde el punto de vista moral", las aberraciones personales, las copias de opiniones de otros seres humanos y las fobias. ${ }^{32}$

Los principios democráticos a los que llegan las sociedades por mutuo consenso, cuyo propósito es regular la conducta de sus miembros según sus prescripciones, no pueden limitar la libertad individual de estos, ya que pueden fundarse en posiciones producidas por prejuicios. Sobre este aspecto, la posición de Habermas es clara en determinar la imposibilidad de una unión de criterios morales.

Para Habermas, la unificación de criterios morales comunes es una utopía, debido a que el sistema de creencias de la conciencia social pudo basarse en principios tradicionales que, con el paso del tiempo, fueron superados. Este hecho genera una marcada influencia del tradicionalismo que impide el acoplamiento con nuevas concepciones morales y la imposibilidad de una descripción real de la sociedad. Respecto a esta problemática, es necesario hacer una descripción externa y la posterior aplicación de patrones recurrentes en el sistema social; este proceso se llama colonización de la vida. ${ }^{33}$

Este proceso sistematiza lo que está implicado dentro del mundo de la vida; así, de manera paulatina, se tiende a la juridización de los sistemas sociales, con el fin de conquistar este mundo; además, el hecho de judicializar los elementos del mundo de la vida para integrarlos a un sistema organizado permite reemplazar la obsoleta teoría de la conciencia de clase. Sin embargo, la penetración del Derecho en el mundo de la vida y en su proceso de axiomatización de instituciones sociales desemboca en un aumento considerable de normas escritas que abarcan dos vías: 1) extensión del Derecho, y 2) adensamiento del Derecho. La primera alude a la regulación de nuevos asuntos que antes no estaban reglados en lo jurídico y, antes de ser axiomatizados, solo eran tratados de modo informal; el segundo término hace referencia a la posibilidad de que una materia jurídica general puede subdividirse en materias jurídicas específicas para facilitar su entendimiento. ${ }^{34}$

\footnotetext{
Dworkin, Los derechos en serio. 366.

Jurgen Habermas, Teoría de la acción comunicativa (Madrid: Trota, 2010), 502.

Habermas, Teoría de la acción comunicativa, 503-506.
}

NOVUM JUS • ISSN: 1692-6013 • Volumen 9 Nº 1 • Enero - Junio 2015 • Págs. 29-49 
La labor del legislador como ente encargado de la axiomatización de conductas para integrarlas a un sistema jurídico debe ser en extremo escrupulosa, ya que a la hora de adoptar un consenso moral como fundamentación de la expedición de una ley, debe examinar el consenso con mucho cuidado. La reacción de la comunidad ante alguna práctica desfavorecida, la percepción de los fundamentos de la reacción negativa que tiene la comunidad frente al acto, etc., son algunas herramientas útiles que deberá tener en cuenta el legislador a la hora de prohibir y sancionar una acción humana; de ese modo, tendrá mayor claridad a la hora de distinguir cuáles son teorías argumentativas válidas, posiciones morales válidas y cuáles son prejuicios, fobias y racionalizaciones. Es posible que, después de dicho procedimiento, no se demuestre la existencia real de un consenso moral, ${ }^{35}$ como se ilustra en el siguiente gráfico:

Gráfico 1.

Proceso de asimilación de la moral convencional al sistema jurídico

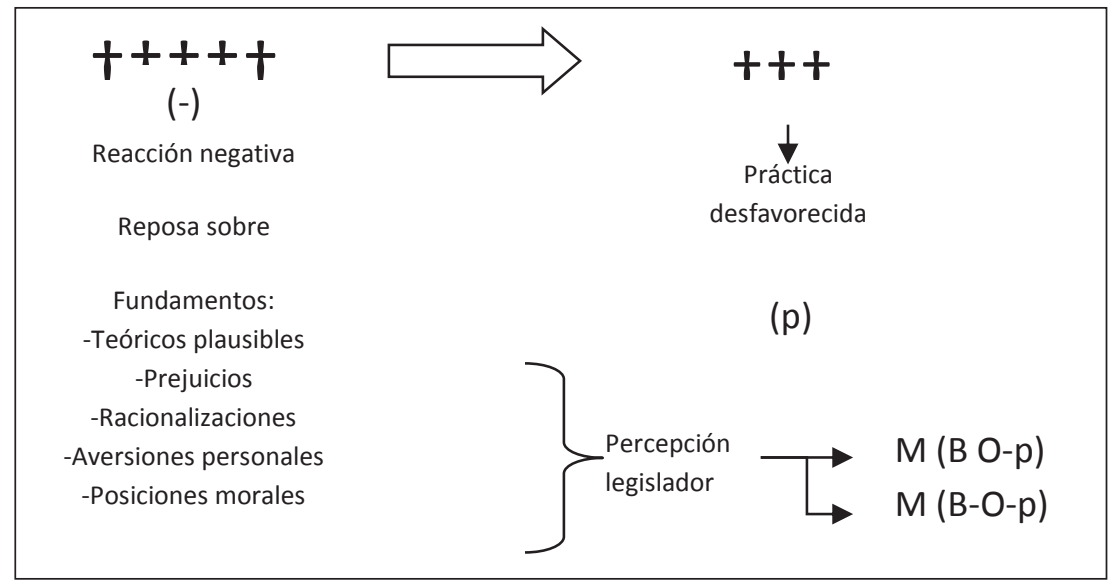

Fuente: elaboración propia.

Al tener en cuenta lo expuesto antes es preciso discutir las posturas existentes frente al fenómeno de la pornografía. Son varios los argumentos esgrimidos en contra de ella; uno es que incita al consumidor a perpetrar delitos sexuales. Si bien es cierto que algunos de los consumidores más asiduos de pornografía han cometido

35 Dworkin, Los derechos en serio, 367. 
crímenes contra la libertad sexual de las personas, no es cierto que se presente en todos los casos; entonces, la incidencia de contenido erótico de la pornografía no es determinante en la conducta criminal de un ser humano. Otro argumento es la necesidad de limitar la libertad de los productores, actores y editores de este tipo de material. La sociedad tiene derecho de protección ante dichas personas, siempre y cuando lo que ellas busquen sea la inmoralidad. De este argumento se puede interpretar que todo material que tenga fines diversos al erotismo es aceptado desde la moral, dentro de un criterio sexual "educativo", pero si por el contrario solo tiene como fin la lujuria, se establecen límites que resultarán del consenso público, mas no de la adhesión a posturas éticas. ${ }^{36}$

La libertad como principio solo puede limitarse desde verdaderas convicciones morales públicas, resultantes del consenso de las posiciones morales válidas e individuales; si, por alguna razón, la aberración a la pornografía es fruto, prejuicio o racionalización, el sistema jurídico queda imposibilitado para limitar la libertad de estos individuos.

Lo curioso de la pornografía es la variedad de puntos de vista sobre el tema, ya que evidencia una doble moral. Primero, en la mayoría de este material se llevan a cabo prácticas eróticas explícitas como sodomía, humillaciones, sadismo, fornicación, etc., que son recibidas por cualquier consumidor; sin embargo, cuando se pregunta a este consumidor lo que piensa sobre la sodomía y el sexo sádico, argumenta que son inmorales y que no deberían presentarse en una sociedad decente. Si tomáramos esta postura como válida desde la moral, ¿no se estaría incurriendo en una contradicción argumentativa de nuestras creencias?, es decir, bajo esta concepción, es moral disfrutar de cualquier tipo de aberración sexual "es más, deleitarse" y, a la vez, rechazar cualquier acto que se le parezca. En términos lógicos, esta aparente posición moral es paradójica, pues no está bien fundamentada: iserá que si dicha persona tiene la oportunidad propicia hará realidad estas fantasías sexuales que ve en los productos pornográficos?

\section{Conclusiones}

El proceso de axiomatización por parte del sistema jurídico de conductas proscritas por la moralidad pública convencional es posible; no obstante, demanda

36 Dworkin, Los derechos en serio, 371.

NOVUM JUS • ISSN: 1692-6013 • Volumen 9 Nº 1 • Enero - Junio 2015 • Págs. 29-49 
de la autoridad nomotética un análisis concienzudo de las racionalizaciones, de las fobias, de reacciones y de teorías plausibles que alimentan la percepción del problema; de ese modo, el legislador adquirirá criterios argumentativos racionales para que, por medio del proceso de valuación normativa, transforme el estatus de un radical deóntico por el de una norma jurídica genuina. En otras palabras, el radical deóntico es asimilado por el Derecho para que sea parte de su integralidad.

La moralidad pública convencional no es un concepto estático, permanente y ahistórico, puesto que, al funcionar como un sistema regulador de conducta humana que funciona paralelamente al Derecho, requiere una capacidad de adaptabilidad. Este requisito de existencia se debe a que el comportamiento de las sociedades humanas es cambiante; entonces, un sistema regulado de conductas que no se acople a la naturaleza dinámica del ser humano está destinado a desaparecer por su paulatina inaplicabilidad.

La partícula esencial de toda moralidad pública convencional es la posición moral individual; esta posición subjetiva puede ser calificada como irracional e inválida a partir de la aplicación de los cuatro requisitos argumentativos desarrollados por Dworkin. A consecuencia de ello, un sistema de creencias que no cumpla con dichas exigencias es calificado como producto de racionalización, prejuicios o fobias; asimismo, no debe ser atendido por la sociedad y mucho menos por un órgano emisor de normas jurídicas.

Debido a la pluralidad cultural, religiosa e ideológica "típica de las sociedades modernas", coexisten posiciones morales válidas incompatibles entre sí; desde esta óptica se construye una moral convencional real y no una de mayorías. Este proceso constructivo requiere el entrelazamiento de los puntos de coincidencia de las posturas morales en una red, que sustentará las razones y motivaciones de las prohibiciones de conductas inmorales.

\section{Referencias}

Austin, John. El objeto de la jurisprudencia. Madrid: Centro de Estudios Políticos y Constitucionales, 2002.

Dworkin, Ronald. Los derechos en serio. Barcelona: Ariel, 2007.

Echave Delia, Ricardo Guibourg y María Urquijo. Lógica, proposición y norma. Buenos Aires: Astrea, 2008. 
Gianella de Salama, Alicia y Margarita Roulet. Lógica simbólica y elementos de metodología de la ciencia. Buenos Aires: El Ateneo, 1986.

Habermas, Jurgen. Teoría de la acción comunicativa. Madrid: Trota, 2010.

Hart, Herbert. El concepto del Derecho. Buenos Aires: Abeledo-Perrot, 1968.

Kant, Immanuel. La metafísica de las costumbres. Madrid: Tecnos, 2002.

Kelsen, Hans. Teoría pura del Derecho. México D. F: Dirección de publicaciones UNAM, 1986.

Nino, Carlos. Introducción al análisis del Derecho. Barcelona: Ariel, 1983.

Santo Tomás de Aquino. La suma de Teología II parte I-II. Madrid: Biblioteca de autores cristianos, 1993.

Schmill, Ulises. Lógica y Derecho. México D. F.: Fontamara, 2008.

Von Wright, Georg. Normas, verdad y lógica. México D. F.: Fontamara, 2001.

NOVUM JUS • ISSN: 1692-6013 • Volumen 9 Nº 1 • Enero - Junio 2015 • Págs. 29-49 
